\title{
Relapse of Mania due to Sleep Deprivation: A Case Report
}

\author{
Vineet Jalota ${ }^{1}$, Swarna B Nayok ${ }^{2}$, T Sathyanarayana Malleswaram ${ }^{3}$, HS Akshatha ${ }^{4}$
}

\begin{abstract}
Background: Sleep disturbances in bipolar disorder exert negative impact. Here we report a case of bipolar disorder, who had manic relapse due to decreased sleep because of waking up early during religious festivities.

Case description: Mrs S is a 50-year-old married Muslim lady was diagnosed to have bipolar affective disorder (BPAD) for the past 25 years. Till now, she had about 8 to 10 manic episodes. From December 2017, she has remained euthymic, being compliant on tab. lithium $1200 \mu \mathrm{g} / \mathrm{day}$ (serum lithium level: $0.79 \mathrm{mmol} / \mathrm{L}$ ). During her scheduled follow-up (May 25, 2019), she reported decreased need for sleep, grandiose ideas, increased psychomotor activity and energy from the start of Ramadan, about 20 days ago. During these 20 days, she woke up about 2 hours earlier to prepare food for family. She was compliant on lithium. Her Young Mania Rating Scale (YMRS) score on the first day was 19, showing

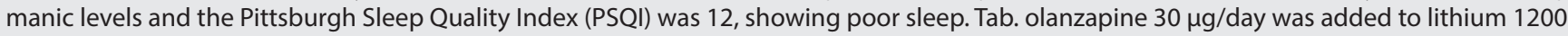
$\mu \mathrm{g} /$ day. She showed gradual improvement in quality and quantity of sleep and was discharged after 2 weeks (June 09, 2019), with tab. lithium $1200 \mu \mathrm{g} /$ day and tab. olanzapine $10 \mu \mathrm{g} /$ day. The patient and her family were psychoeducated regarding the importance of sleep and compliance. During the next follow-up after 5 days, she showed improvements.

Conclusion: Sleep architecture changes in rapid eye movement (REM) sleep like shortened latency and increased density may contribute toward hypomanic/manic symptoms. In bipolar disorder, sleep disturbances even on one previous night may subsequently change the mood toward mania, in spite of having adequate serum lithium levels.

Clinical significance: Psychiatrists need to be vigilant regarding this and provide psychoeducation on sleep hygiene especially with sleep deprivation.
\end{abstract}

Keywords: Bipolar disorder, Mania, Relapse, Sleep deprivation.

Indian Journal of Sleep Medicine (2020): 10.5005/jp-journals-10069-0047

\section{BACKGROUND}

Sleep disturbances such as decreased overall sleep hours and quality during interepisodic period in patients with bipolar affective disorder (BPAD) may precipitate a manic or hypomanic episode. Any change in the circadian rhythm and sleep-wake cycle and quantity and quality of sleep can also incite such events. ${ }^{1}$ Here we report a case of bipolar disorder who had manic relapse due to decreased quality and hours of sleeping due to religious festivities.

\section{Case Description}

Mrs S. is a 50-year-old married Muslim lady. She is a homemaker and lived in a nuclear family at a semiurban town. She was diagnosed to have BPAD for the past 25 years. Till now, she has had about 8 to 10 manic episodes, all without psychotic symptoms. From 2018, she has been receiving treatment from us. Her last episode of mania was in December 2017 and was treated with tab. olanzapine 10 $\mu \mathrm{g} /$ day, gradually tapered and tab. lithium carbonate $1200 \mu \mathrm{g} / \mathrm{day}$. From January 2018, she remained euthymic, being compliant on tab. lithium $1200 \mu \mathrm{g} /$ day (serum lithium level: $0.79 \mathrm{mmol} / \mathrm{L}$ ). During her last scheduled follow-up (May 25, 2019), she reported decreased need for sleep, grandiose ideas, and increased psychomotor activity and energy. These symptoms were from the start of Ramadan, about 20 days ago. In these 20 days, she woke up about 2 hours earlier, at around 4 A.M. to prepare food for her family. She used to go to sleep as usual at about 11 P.M. at night. She was compliant on lithium during this time. Her Young Mania Rating Scale (YMRS) score on the first day was 19, showing manic levels, and her Pittsburgh Sleep Quality Index (PSQI) was 12, showing poor sleep in the last 20 days. Tab. olanzapine $30 \mu \mathrm{g} /$ day was added to lithium $1200 \mu \mathrm{g} /$ day. She showed gradual improvement in quality and quantity of

\footnotetext{
${ }^{1-4}$ Department of Psychiatry, Sri Siddhartha Medical College and Hospital, Tumkur, Karnataka, India
}

Corresponding Author: T Sathyanarayana Malleswaram, Department of Psychiatry, Sri Siddhartha Medical College and Hospital, Tumkur, Karnataka, India, e-mail: sanamathi23@gmail.com

How to cite this article: Jalota V, Nayok SB, Malleswaram TS, et al. Relapse of Mania due to Sleep Deprivation: A Case Report. Indian J Sleep Med 2020;15(1):15-16.

Source of support: Nil

Conflict of interest: None

sleep and was discharged after 2 weeks (June 9, 2019), with tab. lithium $1200 \mu \mathrm{g} /$ day and tab. olanzapine $10 \mu \mathrm{g} /$ day. On discharge day, her YMRS score was 5 and PSQI score was 4, both showing significant improvement. Reviewing her previous episodes, we found that two of her previous manic episodes too coincided with the month of Ramadan, in August 2011 and May 2017. Family and patient were psychoeducated regarding the importance of sleep and compliance. During the next follow-up after 5 days, her YMRS score was 4 and PSQI was 3, showing maintained improvement. Since then she has been maintaining well and is on monthly regular follow-up, i.e., on $1200 \mu \mathrm{g}$ of tab. lithium carbonate per day, with a serum lithium level of $1.28 \mathrm{mmol} / \mathrm{L}$.

\section{Discussion}

Sleep architecture changes like REM sleep, shortened REM latency, and increased REM density contribute toward hypomanic/manic symptoms. In BPAD, sleep disturbances even on one previous night may subsequently change the mood toward mania. ${ }^{1}$ Changes in 
social rhythms or circadian rhythms due to any reason, for example, Ramadan in our case, have been attributed as one of the reasons for relapse of mania. ${ }^{2}$ Dehydration may result in lithium toxicity, but relapses due to variations in serum lithium levels owing to dehydration have not been reported. ${ }^{3}$ Reviews on health effects of Ramadan found that in general population no harms on physical health were attributed to Ramadan. ${ }^{4-6}$ It has also shown to have an overall positive effect on mental health. ${ }^{7,8}$ However, there may be more patients with alcohol withdrawal due to restriction in alcohol use during longer fasting periods and deterioration in psychopathology in patients with schizophrenia with metabolic syndrome have been noted before. ${ }^{9,10}$ Although general mood of patients with mood disorder improves, relapses in mood disorder may also increase, owing to fasting..$^{2-4}$ Our patient had no signs of dehydration and had adequate lithium levels as shown by serum lithium levels before the relapse of mania. Thus, we consider sleep disturbance as the main reason for relapse.

\section{Conclusion}

Sleep deprivation due to various reasons which may include religious festivities may cause relapse or precipitation of manic or hypomanic symptoms in those suffering from bipolar illness, in spite of having adequate serum lithium levels.

\section{Clinical Significance}

Psychiatrists need to be vigilant regarding this and provide psychoeducation on sleep hygiene and treatment compliance especially in situations which cause fasting and sleep deprivation.

\section{References}

1. Eidelman P, Talbot LS, Gruber J, et al. Sleep architecture as correlate and predictor of symptoms and impairment in inter-episode bipolar disorder: taking on the challenge of medication effects. J Sleep Res 2010;19(4):516-524. DOI: 10.1111/j.1365-2869.2010.00826.x.

2. Eddahby S, Kadri N, Moussaoui D. Ramadan and bipolar disorder: example of circadian rhythm disturbance and its impact on patients with bipolar disorders. L'Encephale 2013;39(4):306-312. DOI: 10.1016/ j.encep.2012.11.008.

3. Eddahby S, Kadri N, Moussaoui D. Fasting during Ramadan is associated with a higher recurrence rate in patients with bipolar disorder. World Psychiatry. 2014;13(1):97-97. DOI: 10.1002/wps.20113.

4. Heun R. A systematic review on the effect of Ramadan on mental health: minor effects and no harm in general, but increased risk of relapse in schizophrenia and bipolar disorder. Glob Psychiatry. 2018;1(1):7-16.

5. Rouhani MH, Azadbakht $\mathrm{L}$. Is Ramadan fasting related to health outcomes? A review on the related evidence. J Res Med Sci Off J Isfahan Univ Med Sci 2014;19(10):987-992.

6. Adawi M, Watad A, Brown S, et al. Ramadan fasting exerts immunomodulatory effects: insights from a systematic review. Front Immunol [Internet] 2017;8:1144. DOI: 10.3389/ fimmu.2017.01144Available from: https://www.frontiersin.org/ articles.

7. Roky R, Iraki L, HajKhlifa R, et al. Daytime alertness, mood, psychomotor performances, and oral temperature during ramadan intermittent fasting. Ann Nutr Metab 2000;44(3):101-107. DOI: 10.1159/000012830.

8. Nugraha B, Ghashang SK, Hamdan I, et al. Effect of ramadan fasting on fatigue, mood, sleepiness, and health-related quality of life of healthy young men in summer time in Germany: a prospective controlled study. Appetite. 2017;111:38-45. DOI: 10.1016/j.appet.2016.12.030.

9. Fawzi MH, Fawzi MM, Said NS, et al. Effect of Ramadan fasting on anthropometric, metabolic, inflammatory and psychopathology status of Egyptian male patients with schizophrenia. Psychiatry Res. 2015;225(3):501-508. DOI: 10.1016/j.psychres.2014.11.057.

10. Al-Sinawi $\mathrm{H}, \mathrm{Al}-$ Adawi $\mathrm{S}$, Al-Guenedi A. Ramadan fasting triggering koro-like symptoms during acute alcohol withdrawal: a case report from Oman. Transcult Psychiatry. 2008;45(4):695-704. DOI: $10.1177 / 1363461508100791$. 